\title{
Nonadiabatic pairing effects for tight-binding electrons interacting with phonons
}

\author{
A. Perali ${ }^{1}$, C. Grimaldi ${ }^{1,2}$ and L. Pietronero ${ }^{1,2}$ \\ ${ }^{1}$ Dipartimento di Fisica, Universitá di Roma "La Sapienza", Piazzale A. Moro, 2, 00185 Roma, Italy \\ ${ }^{2}$ Istituto Nazionale Fisica della Materia, Unitá di Roma 1, Italy
}

(August 13, 2018)

\begin{abstract}
The nonadiabatic electron-phonon corrections for the superconducting pairing are investigated for a specific tight-binding model corresponding to a $2 d$ square lattice. This permits to investigate the role of various specific properties like the band filling, nesting effects and a realistic van Hove singularity on the superconducting effective pairing beyond Migdal's limit. The main results are: (i) Starting from a momentum independent electron-phonon coupling the nonadiabatic effects lead to an effective pairing which is strongly dependent on frequency and momentum. (ii) If instead the electron-phonon coupling is mainly forward (as due to correlation effects) the resulting pairing results to be strongly enhanced. These results confirm but also extend the simplified scheme used up to now to compute these properties. In this respect our results link the nonadiabatic effects to the specific properties of realistic materials.

PACS numbers:74.20.Mn, 71.38.+i, 63.20.Kr
\end{abstract}

\section{INTRODUCTION}

Unconventional superconductors, such as guprates and fullerides, are characterized by Fermi energies $E_{F}$ much smaller than those of conventional metals. 3 In this situation, the energy scale $\omega_{0}$ of the mediator of the superconducting pairing can be comparable to $E_{F}$ so that the quantity $\omega_{0} / E_{F}$ is no longer negligible as it happens for conventional superconductors. As a consequence, vertex corrections in the normal state electronic self-energy become relevant because of the non-validity of Migdal's approximationl and also the Migdal-Eliashberg (ME) approach to the

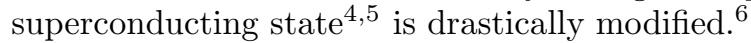

In recent years we have generalized both the superconducting and the normal states in order to include the first corrections beyond Migdal's limit. 11 We considered the energy scale $\omega_{0}$ to be given by phonons so that the parameter $\omega_{0} / E_{F}$ measures the degree of adiabaticity of the lattice dynamics compared to the electronic kinetic energy. For $\omega_{0} / E_{F} \ll 1$ and electron-phonon $(e-p h)$ coupling $\lambda<1$ one recovers the ME theory of the electron-phonon coupled system. By enhancing the coupling $\lambda$ the electron develops a large effective mass (polaron) and eventually forms bi-polaronic states. However, the $e-p h$ coupled system may display a regime which is different from both the ME picture and the polaronic (bi-polaronic) regime. We have named this situation nonadiabatic, and it is characterized by quasi-free electrons, i.e., $\lambda<1$, coupled in a nonadiabatic way $\left(\omega_{0} / E_{F}\right.$ not negligible) to the lattice. We have shown that in this regime it is possible to define a perturbative theory where the expansion parameter is roughly given by $\lambda \omega_{0} / E_{F}$. Moreover, the corrections arising from the nonadiabatic hypothesis have a non-trivial structure in both the momentum transfer $q$ and the Matsubara exchanged frequency $\omega$. In fact, the vertex correction appearing in the normal state self-energy becomes positive (negative) when $v_{F} q<\omega\left(v_{F} q>\omega\right)$, where $v_{F}$ is the Fermi velocity.8 The generalization to the superconducting transition reveals that this situation is also encountered for the class of diagrams beyond Migdal's limit relevant for the Cooper channel. Concerning the critical temperature $T_{c}$, as long as the momentum transfer is less than $\omega_{0} / h^{k}$ the nonadiabatic corrections lead to a strong enhancement of $T_{c}$ also for moderate values of the e-ph coupling $\lambda .6 \mathrm{E}$ The opening of a superconducting gap changes drastically the behavior in momentum-frequency space of the vertex especially in the low temperature limit. In this regime the vertex can be positive also when $v_{F} q>\omega$ probably leading to anomalous values of the gap-to- $T_{c}$ ratio. 11

The results presented in the previous papers on the nonadiabatic e-ph interaction theory have been derived by employing higly simplified approximations. Although several results were approximation-independent, it is important to study the behavior of the nonadiabatic corrections by using realistic models. The aim of this paper is to provide a study of both the vertex and cross diagrams in the framework of a specific tight-binding model for the electronic band. The advantage of such a study is twofold: it permits to confirm the results obtained by using simple models but also the whole picture is made much richer so that it is possible to relate specific nonadiabatic properties to specific features of the material. Finally, this paper can be also regarded as a preliminary work for a completely numerical solution of the Eliashberg equations generalized beyond Migdal's limit.

The paper is organized as follows. In Sec. II we introduce the equations for the superconducting instability driven by the $e-p h$ coupling generalized in order to include the first nonadiabatic corrections represented by the vertex and cross diagrams for a specific tight-binding model. In Sec.III we study the vertex and cross corrections for a model in which the $e-p h$ interaction is momentum independent while in Sec.IV we analyze the effect on the nonadiabatic 
corrections of an $e$ - $p h$ coupling peaked at small values of the momentum transfer as induced by electronic correlations. The last section is devoted to the conclusions.

\section{BEYOND MIGDAL-ELIASHBERG THEORY}

The presence of nonadiabatic corrections beyond Migdal's limit affects both the normal and superconducting properties of the metallic systems in a non-trivial way. In fact, as it has been already shown in Refs. 7, 8, up to the first corrections of order $\lambda \omega_{0} / E_{F}$, the normal state electronic self-energy is modified by only one nonadiabatic correction, the vertex correction, while for the superconductive instability one needs to include also the cross correction. This situation is shown in Fig.1 where the normal state electronic self-energy $\Sigma_{N}$ (Fig.1 1 a) and the linearized gap equation (Fig.1 $\mathrm{b}$ ) are depicted in order to include the first corrections beyond Migdal's limit.

In order to find the critical temperature $T_{c}$, one has to solve the following coupled set of equations relating the diagonal, $\Sigma_{N}(k)$, and the off-diagonal, $\Sigma_{S}(k)$, electron self-energies:

$$
\begin{aligned}
& \Sigma_{N}(k)=\sum_{k^{\prime}} V_{0}\left(k-k^{\prime}\right)\left[1+P\left(k, k^{\prime}\right)\right] G\left(k^{\prime}\right), \\
& \Sigma_{S}(k)=\sum_{k^{\prime}} V\left(k, k^{\prime}\right) G\left(k^{\prime}\right) G\left(-k^{\prime}\right) \Sigma_{S}\left(k^{\prime}\right),
\end{aligned}
$$

where $k=\left(i \omega_{k}, \mathbf{k}\right), k^{\prime}=\left(i \omega_{k^{\prime}}, \mathbf{k}^{\prime}\right)$, and we use the notation $\sum_{k}=-T_{c} \sum_{\omega_{k}} \sum_{\mathbf{k}}$. In the above equations, $V_{0}\left(k-k^{\prime}\right)=$ $g\left(\mathbf{k}-\mathbf{k}^{\prime}\right)^{2} D\left(k-k^{\prime}\right)$ where $g\left(\mathbf{k}-\mathbf{k}^{\prime}\right)$ is the $e$-ph matrix element, $G(k)$ and $D(q)$ are the electron and phonon propagators given by:

$$
G^{-1}(k)=i \omega_{k}-\epsilon(\mathbf{k})-\Sigma_{N}(k)
$$

and

$$
D(q)=-\frac{\omega_{0}^{2}}{\omega_{q}^{2}+\omega_{0}^{2}}
$$

respectively. In equations (3) and (4), $\epsilon(\mathbf{k})$ is the electronic band dispersion and $\omega_{0}$ is the phonon frequency which we assume dispersionless for simplicity. In Eq.(1) $P\left(k, k^{\prime}\right)$ is the first vertex correction beyond Migdal's limit, which according to Fig. 17a is given by:

$$
P\left(k, k^{\prime}\right)=\sum_{p} V_{0}(k-p) G\left(p-k+k^{\prime}\right) G(p) .
$$

The total pairing interaction $V\left(k, k^{\prime}\right)$ appearing in the linearized gap equation (2) is instead given by:

$$
V\left(k, k^{\prime}\right)=V_{0}\left(k-k^{\prime}\right)\left[1+2 P\left(k, k^{\prime}\right)\right]+C\left(k, k^{\prime}\right)
$$

where $P\left(k, k^{\prime}\right)$ is again the vertex correction (5) while $C\left(k, k^{\prime}\right)$ is the cross term (last diagram appearing in Fig.11b):

$$
C\left(k, k^{\prime}\right)=\sum_{p} V_{0}(k-p) V_{0}\left(p-k^{\prime}\right) G(p) G\left(p-k-k^{\prime}\right) .
$$

The ordinary Migdal-Eliashberg equations are obtained by neglecting both the vertex $P$ and cross $C$ corrections in Eqs.(1):2.6), and in this limit the pairing interaction (6) reduces to $V_{0}\left(k-k^{\prime}\right)$.

From the above equations, it is clear that to obtain the diagonal and off-diagonal self-energies one has to calculate the vertex and cross correction $P\left(k, k^{\prime}\right.$ and $C\left(k, k^{\prime}\right)$. In previous papers we have presented the first detailed evaluations of these corrections both for 3 -d 0 and 2 -d systems 9 However in calculating $P$ and $C$ we have employed several approximations: the most important ones are probably the linearization of the electronic dispersion and the assumption of half filling. Although the former approximation is valid for small momentum transfer in the $e$-ph scattering, it becomes poor for momentum transfer of order $k_{F}$. The previous calculations moreover did not address the problem of the role of band filling, which we know to be important for the nonadiabatic corrections. In fact, it has been shown that the hole-particle contribution present in the vertex correction is due to the exchange part of the phonon-mediated electron-electron interaction which strongly depends on the electron density.12 
In this paper we present results obtained by numerical integration of Eqs(5,7) over the Brilloiun zone by using a two dimensional electronic dispersion $\epsilon(\mathbf{k})$ with nearest and next-nearest neighbors hopping elements $\left(t, t^{\prime}\right)$ :

$$
\epsilon(\mathbf{k})=-2 t\left[\cos \left(k_{x}\right)+\cos \left(k_{y}\right)\right]+4 t^{\prime} \cos \left(k_{x}\right) \cos \left(k_{y}\right)-\mu,
$$

where $\mu$ is the chemical potential. The above electronic dispersion is quite general for $2 D$ transport in strongly correlated systems and if -is also suitable to describe the conduction band associated with the $\mathrm{CuO}_{2}$ planes in the high- $T_{c}$ superconductors. 13

The calculation of the vertex and cross corrections for each $k$ and $k^{\prime}$ provides the effective pairing interaction $V\left(k, k^{\prime}\right)$ appearing in the linearized gap equation (2). We show that $V\left(k, k^{\prime}\right)$ has a non-trivial momentum and frequency dependence which can be in favour of a strongly anisotropic $s$-wave solution of the gap or a $d$-wave symmetry when a repulsive potential is added to the kernel. Our calculations are also aimed to show that the anisotropy provided by the nonadiabatic corrections is an important element for a possible enhancement of the critical temperature $T_{c}$. For this reason, any approximation scheme which neglects such a momentum-frequency interplay easily leads to an underestimation of the nonadiabatic contributions to enhancing $T_{c}$.

In all the calculations presented below, we have neglected the self-energy contribution appearing in Eqs.(5,7) by inserting in the diagrams the bare propagators $G_{0}^{-1}(k)=i \omega_{k}-\epsilon(\mathbf{k})$. In this way, the summation over the Matsubara frequencies can be preformed exactly by using the analytical continuation and contour integrals in the complex $z$-plane (Poisson's formula). After this summation is carried out, the vertex and the cross corrections reduces to:

$$
\begin{aligned}
& P\left(k, k^{\prime}\right)=P\left(\mathbf{k}, i \omega_{k} ; \mathbf{k}^{\prime}, i \omega_{k^{\prime}}\right)=\frac{\omega_{0}}{2} \frac{1}{N} \sum_{\mathbf{p}} \frac{g(\mathbf{k}-\mathbf{p})^{2}}{\epsilon(\mathbf{p})-\epsilon\left(\mathbf{p}-\mathbf{k}+\mathbf{k}^{\prime}\right)-i\left(\omega_{k}-\omega_{k^{\prime}}\right)} \\
& \times\left[\frac{f(\epsilon(\mathbf{p}))+n\left(-\omega_{0}\right)}{\epsilon(\mathbf{p})+\omega_{0}-i \omega_{k}}-\frac{f(\epsilon(\mathbf{p}))+n\left(\omega_{0}\right)}{\epsilon(\mathbf{p})-\omega_{0}-i \omega_{k}}-\frac{f\left(\epsilon\left(\mathbf{p}-\mathbf{k}+\mathbf{k}^{\prime}\right)\right)+n\left(-\omega_{0}\right)}{\epsilon\left(\mathbf{p}-\mathbf{k}+\mathbf{k}^{\prime}\right)+\omega_{0}-i \omega_{k^{\prime}}}+\frac{f\left(\epsilon\left(\mathbf{p}-\mathbf{k}+\mathbf{k}^{\prime}\right)\right)+n\left(\omega_{0}\right)}{\epsilon\left(\mathbf{p}-\mathbf{k}+\mathbf{k}^{\prime}\right)-\omega_{0}-i \omega_{k^{\prime}}}\right] .
\end{aligned}
$$

and

$$
\begin{aligned}
& C\left(k, k^{\prime}\right)=C\left(\mathbf{k}, i \omega_{k} ; \mathbf{k}^{\prime}, i \omega_{k^{\prime}}\right)=\frac{\omega_{0}}{2} \frac{2 \omega_{0}+i\left(\omega_{k}+\omega_{k^{\prime}}\right)}{\left(\omega_{k}-\omega_{k^{\prime}}\right)^{2}+\left(2 \omega_{0}\right)^{2}} \frac{1}{N} \sum_{\mathbf{p}} \frac{g(\mathbf{k}-\mathbf{p})^{2} g\left(\mathbf{p}-\mathbf{k}^{\prime}\right)^{2}}{\epsilon(\mathbf{p})-\epsilon\left(\mathbf{p}-\mathbf{k}-\mathbf{k}^{\prime}\right)-i\left(\omega_{k}+\omega_{k^{\prime}}\right)} \\
& \times\left[\frac{f(\epsilon(\mathbf{p}))+n\left(\omega_{0}\right)}{\left(\epsilon(\mathbf{p})-\omega_{0}-i \omega_{k}\right)\left(\epsilon(\mathbf{p})-\omega_{0}-i \omega_{k^{\prime}}\right)}-\frac{f(\epsilon(\mathbf{p}))+n\left(-\omega_{0}\right)}{\left(\epsilon(\mathbf{p})+\omega_{0}-i \omega_{k}\right)\left(\epsilon(\mathbf{p})+\omega_{0}-i \omega_{k^{\prime}}\right)}\right. \\
& \left.-\frac{f\left(\epsilon\left(\mathbf{p}-\mathbf{k}-\mathbf{k}^{\prime}\right)\right)+n\left(\omega_{0}\right)}{\left(\epsilon\left(\mathbf{p}-\mathbf{k}-\mathbf{k}^{\prime}\right)-\omega_{0}+i \omega_{k}\right)\left(\epsilon\left(\mathbf{p}-\mathbf{k}-\mathbf{k}^{\prime}\right)-\omega_{0}+i \omega_{k^{\prime}}\right)}+\frac{f\left(\epsilon\left(\mathbf{p}-\mathbf{k}-\mathbf{k}^{\prime}\right)\right)+n\left(-\omega_{0}\right)}{\left(\epsilon\left(\mathbf{p}-\mathbf{k}-\mathbf{k}^{\prime}\right)+\omega_{0}+i \omega_{k}\right)\left(\epsilon\left(\mathbf{p}-\mathbf{k}-\mathbf{k}^{\prime}\right)+\omega_{0}+i \omega_{k^{\prime}}\right)}\right] .
\end{aligned}
$$

In the above equations, $f$ and $n$ are fermionic and bosonic distribution functions, respectively. The numerical integrations of (9) and (10) are performed by using a momentum mesh of the Brillouin zone of $N=128 \times 128$ points cheking that increasing $N$ does not change appreciably our results. We evaluate the real and imaginary part of the vertex and cross corrections by making use of two different models for the $e$-ph coupling $g(\mathbf{q})$. In the following we report only the real part if the nonadiabatic corrections, being the corresponding imaginary parts at least one order of magnitude less than the real parts

In the next section we adopt a calculation with a momentum independent coupling $g(\mathbf{q})=g_{0}$. This situation is therefore the one encountered for electrons locally coupled with dispersionless phonons (Holstein model). The effect of the momentum dependence of $g(\mathbf{q})$ on the vertex and cross corrections will be studied in Sec.IV.

\section{MOMENTUM INDEPENDENT ELECTRON-PHONON COUPLING}

When we neglect the momentum dependence of the $e-p h$ coupling $g(\mathbf{q})$ in equations (9) and (10), it turns out that the vertex function $P$ depends only on the momentum transfer $\mathbf{q}=\mathbf{k}-\mathbf{k}^{\prime}$, while the cross function $C$ depends on the total momentum $\mathbf{Q}=\mathbf{k}+\mathbf{k}^{\prime}$. This situation permits us to study the momentum dependence of $P$ and $C$ as a function of $\mathbf{q}$ and $\mathbf{Q}$ spanning the Brilloiun zone, respectively.

In Fig.2 we show the calculated vertex correction $P$ as a function of $\mathbf{q}=\mathbf{k}-\mathbf{k}^{\prime}$ for $t^{\prime}=0$ and different values of the chemical potential $\mu$. The calculation has been performed with a coupling $g_{0}^{2} /(4 t)=1$, a temperature small compared to the phonon frequency $\omega_{0}\left(T / \omega_{0}=0.01\right)$, and the adiabatic parameter $\omega_{0} /(4 t)$ has been set equal to 0.2 . 
The incoming and outcoming frequencies are set equal to $\omega_{k}=\pi T$, that is the lowest fermionic Matsubara frequency, and $\omega_{k^{\prime}}=\omega_{0}$, respectively. At half filling $(\mu=0)$, the vertex shows a strong dependence over the momentum transfer q. A maximum positive value is found at $\mathbf{q}=(0,0)$ (which corresponds to $\mathbf{k}=\mathbf{k}^{\prime}$ ), while a negative minimum is found at $\mathbf{q}=(\pi, \pi)$, which corresponds to the nesting vector. Therefore this calculation confirms the qualitative analytic results obtained in previous works, the vertex function at half filling. By moving away from half filling the momentum dependence is weakened and at the same time the region where the vertex assumes negative values is reduced. For $\mu /(4 t)=-0.9$, near the bottom of the electronic band, the vertex is always positive for the frequencies used in the calculation.

This behavior is remarkable, since it shows that the vertex correction is very sensitive to the band filling and to the distance from the van Hove singularity ( $\mathrm{vHs}$ ). 9 This result can be understood by observing that the strong dependence of $P$ on the electron density is due to many-body effects given by the Pauli exclusion principle. In fact it has been shown that the exchange contribution of the phonon mediated electron-electron interaction is responsible for the negative values assumed by the vertex in Matsubara frequencies. By lowering the electron density, these many-body effects are weakened and the vertex becomes mostly positive.12 Moreover, away from half filling, the effect of the vHs is reduced and the $\mathbf{q}$-dependence is reduced.

Note that in Fig.2 the strongest dependence on band filling is in the region close to the point $\mathbf{q}=(\pi, \pi)$. This behavior is due to nesting effects and disappears when nesting is destroyed. In fact, we show in Fig 3 the behavior of the vertex when a next-nearest hopping amplitude $t^{\prime}$ is introduced. The curves for different values of $t^{\prime}$ have been calculated by setting the chemical potential $\mu$ always coincident with the van Hove singularity at $-4 t^{\prime}$, and the remaining parameters have the same values as in Fig.2. It is clear that nonzero values of $t^{\prime}$ have strong effects around $(\pi, \pi)$ while for other values of $\mathbf{q}$ the dependence of the vertex on $t^{\prime}$ is much weaker. Moreover, by enhancing $t^{\prime}$, the negative peak at $\mathbf{q}=(\pi, \pi)$ is weakened and for $t^{\prime} / t>0.3$ is completely destroyed.

So far, the curves of the vertex have been evaluated for frequencies fixed at $\omega_{k}=\pi T$ and $\omega_{k^{\prime}}=\omega_{0}$. The same values of the incoming and outcoming frequencies have been used in Refs. 7.8 for the analytic formula of the critical temperature $T_{c}$, which is in rather satisfactory agreement with the $T_{c}$ given by a fully numerical solution of the generalized Eliashberg equations. 1 However, other physical quantities besides $T_{c}$ are affected in a different way by the nonadiabatic corrections. For example, the effective electronic mass $m^{*}$ is mostly influenced by the vertex function calculated for values of the exchanged frequency $\omega=\omega_{k}-\omega_{k^{\prime}}$ much lower than $\omega_{0} .10$ Therefore, it is important to analyze the role played by the exchanged frequency. To this end, we have calculated the vertex function $P$ for $\omega_{k}=\pi T$ and different values of $\omega_{k^{\prime}}$. In Fig $₫$ we plot $P$ for $t^{\prime}=0, \mu /(4 t)=-0.1$ and $\omega_{k^{\prime}}=(2 r+1) \pi T$, where the integer number $r$ runs from $r=1$, corresponding to $\omega_{k^{\prime}} \simeq 0.06 \omega_{0}$, to $r=64$ which gives $\omega_{k^{\prime}}=4 \omega_{0}$. For $r=16$ we obtain $\omega_{k^{\prime}}=\omega_{0}$ and it is therefore the same situation as in Fig.2. We notice that by lowering the frequency, the vertex assumes more structure in the momentum transfer $\mathbf{q}=\mathbf{k}-\mathbf{k}^{\prime}$ and the positive peak at $\mathbf{q}=(0,0)$ becomes narrower. This behavigr is consistent with the condition $v_{F}|\mathbf{q}|<\omega$, where $v_{F}$ is the Fermi velocity, for the positivity of the vertex function.

Summarizing the above analysis, the vertex function has a strong momentum-frequency dependence at half filling and it is positive for small momentum transfer. Away from half filling, the dependence on the momentum is weakened, but at the same time the region where the vertex is negative is reduced. It is important to notice that in the linearized gap equation (2) the vertex corrections are always multiplied by the phonon propagator which provides a negative sign (see Eqs. (4.,60). Therefore, positive values of the vertex give attractive contributions to the pairing interaction $V\left(k, k^{\prime}\right)$ and can lead to an enhancement of $T_{c}$.

The question now concerns the behavior of the other nonadiabatic correction appearing in the kernel of the generalized Eliashberg equations, i.e., the cross function $C(10)$. As we have said at the beginning of this section, when the $e-p h$ coupling is momentum independent, the cross function depends on the external momenta $\mathbf{k}$ and $\mathbf{k}^{\prime}$ only through the total momentum $\mathbf{Q}=\mathbf{k}+\mathbf{k}^{\prime}$. This momentum dependence is quite different from the one relevant for the vertex correction $\left(\mathbf{q}=\mathbf{k}-\mathbf{k}^{\prime}\right)$. However, the results for the cross are qualitatively the same of those for the vertex provided we change $\mathbf{q}$ in $\mathbf{Q}$. In fact, we show in Fig. 5 the cross correction in units of $4 t$ as a function of $\mathbf{Q}=\mathbf{k}+\mathbf{k}^{\prime}$ for different fillings. As in Fig.2, we set $t^{\prime}=0, \omega_{0} /(4 t)=0.2, \omega_{k}=0$ and $\omega_{k^{\prime}}=\omega_{0}$. At half filling $(\mu=0)$, the cross function is attractive for $\mathbf{Q}=(0,0)$ and repulsive at $(\pi, \pi)$. Away from half filling, the $\mathbf{Q}$-dependence is weakened and for $\mu /(4 t)=-0.9$ the cross remains always attractive. As for the vertex function, the feature at $\mathbf{Q}=(\pi, \pi)$ at half filling is given by nesting effects. These effects are removed for $t^{\prime} \neq 0$ as it is shown in Fig.6 where the chemical potential is set equal to the vHs energy $\left(\mu=-4 t^{\prime}\right)$.

From the above discussion, the situation for the effective pairing interaction $V\left(k, k^{\prime}\right)$, Eq.(同), appearing in the linearized gap equation (2) is the following. The contributions coming from the vertex depend on $\mathbf{q}=\mathbf{k}-\mathbf{k}^{\prime}$ and, near half filling, they are attractive for $\mathbf{q} \simeq(0,0)$ while the cross term is attractive for $\mathbf{Q}=\mathbf{k}+\mathbf{k}^{\prime} \simeq(0,0)$. The structure in the momenta of the effective interaction is therefore nontrivial because of the differences between the vertex and cross dependence over momenta.

In this situation, the momentum dependence of the pairing interaction $V\left(k, k^{\prime}\right)$ is better described by employing 
the angular representation shown in fig.7 and described as follows. We consider both the momenta on the Fermi surface $\mathbf{k}=\mathbf{k}_{F}$ and $\mathbf{k}^{\prime}=\mathbf{k}_{F}^{\prime}$ and we define the angle $\phi$ between these vector momenta with respect to the center of the closed holes Fermi surface centered around the $M=(\pi, \pi)$ point of the Brillouin zone. The momentum $\mathbf{k}_{F}$ is then fixed each times on relevant few points and $\mathbf{k}_{F}^{\prime}$ runs on the whole Fermi surface taking into account small and large $\mathbf{q}$ scattering in all directions. This angular representation is also very useful when we shall study in the next section the effects of the momentum-dependence of the $e$ - $p h$ interaction.

The resulting angular representation of the vertex $P$, the cross $C$ and the total pairing interaction $V$ is shown in Fig.8. The calculation has been performed with the same set of values as in Fig. 2 with the chemical potential $\mu /(4 t)=0.1$, i.e., slightly above the van Hove singularity corresponding to an open electron Fermi surface (closed holes Fermi surface centered around $M=(\pi, \pi))$. The momentum $\mathbf{k}_{F}$ is fixed at $\left(k_{x F}, \pi\right)$ near the saddle point located at $Y=(0, \pi)$ where the local density of states $n(\mathbf{k})=1 /|\nabla \epsilon(\mathbf{k})|(L D O S)$ diverges. The vertex $P$ has the maximum positive value for $\phi=0(\mathbf{q}=(0,0))$ corresponding to an attractive contribution for $2 V_{0}\left(k-k^{\prime}\right) P\left(k, k^{\prime}\right)$ at $\phi=0$; the cross $C$ has the minimum negative value for $\phi=\pi(\mathbf{Q}=(0,0))$ and a small local minimum negative value for $\phi=0\left(\mathbf{Q}=\left(2 k_{x F}, 2 \pi\right)\right)$. The total effective interaction $V$, Eq.(6), acquires therefore a strong momentum dependence ( $\phi$-dependence) solely due to the nonadiabatic corrections. Compared to the bare structureless interaction $V_{0}$, the effective pairing $V$ is enhanced at $\phi \simeq 0$ (due to vertex contributions) and at $\phi \simeq \pm \pi$ (due to the cross contribution). Note that $V$ is weakened at $\phi \simeq \pm \pi / 2$ with respect to $V_{0}$. These results lead to the following qualitative consideration. If we imagine to add a repulsive interaction $U$ chosen in such a way that, for frequencies of order $\omega_{0}, U+V_{0} \simeq 0$, the inclusion of the nonadiabatic corrections leads to the total effective interaction $U+V \simeq 2 V_{0} P+C$ which has a momentum dependence consistent with a $d$-wave like symmetry of the gap parameter.

The result of this section is that the vertex and cross corrections have structures which give attractive interaction at different point of the Brillouin zone. It has to be stressed that, although we start with a momentum independent e-ph interaction $g(\mathbf{q})=g$, the nonadiabatic corrections give rise to a strong momentum dependence of the kernel for the superconducting instability. In view of the present analysis, it appears therefore that in calculating the critical temperature $T_{c}$ beyond Migdal's limit one has to deal with the strong frequency-momentum dependence of the nonadiabatic corrections. For example, In Ref. 14 the authors employ an approximation scheme which automatically neglects the momentum-frequency interplay of the vertex and cross corrections and this leads to a reduction of the critical temperature $T_{c}$.

We will see in the following section, that when we consider a model for which the $e-p h$ coupling has peaked structure for small momentum transfer, the nonadiabatic corrections, and in particular the cross function, change drastically their momentum dependence with respect to the case studied in this section.

\section{SMALL $Q$ ELECTRON-PHONON COUPLING}

We consider in this section the momentum dependence of the $e-p h$ coupling $g(\mathbf{q})$ using again the tight-binding electron dispersion (8) and in particular we study the small $q$ limit corresponding to almost forward scattering or long wavelenght phonons. Attractive effective couplings peaked at small momenta are naturally obtained in models with strong local repulsive term-and short range attractive terms such as Hubbard-Holstein model, $t-J$ models and three bands Hubbard model.15 17 All these models show phase separation instability signalled by divergent scattering amplitude at zero momenta; near phase separation the large charge fluctuations can mediate the $e-e$ interactions and give rise to pairing in the Cooper channel with $d$-wave or strongly anisotropic $s$-wave symmetry of the gap parameter 18 An e-ph coupling peaked at small moment $u m$ transfer can also be the consequence of weak screening effects in a system with small charge carrier density. 1921

In our analysis we consider the following form for the $e$-ph coupling $g(\mathbf{q})$

$$
g(\mathbf{q})^{2}=\frac{\gamma\left(q_{c}\right)}{q_{c}^{2}+\omega(\mathbf{q})^{2}},
$$

where

$$
\omega(\mathbf{q})^{2}=2\left[2-\cos \left(q_{x}\right)-\cos \left(q_{y}\right)\right] .
$$

This coupling has the periodicity of the lattice and, for small values of the momentum transfer $\mathbf{q}, \omega(\mathbf{q})^{2} \simeq|\mathbf{q}|^{2}$ so that $g(\mathbf{q})^{2}$ reduces to a lorentzian form. In this way it is easy to understand the role played by the parameter $q_{c}$ : it provides a momentum cutoff in the $e-p h$ interaction in such a way that for $|\mathbf{q}|>q_{c}$ the coupling is depressed. Therefore the coupling (11) and (12) reproduces qualitatively the momentum dependence of the $e$-ph interaction in the presence of strong electronic correlation. 
In order to compare the results on the nonadiabatic corrections calculated by using the model (11) and (12) with the structureless $e-p h$ coupling case of the previous section, we introduce the quantity $\gamma\left(q_{c}\right)$ defined in order to satisfy the following normalization condition:

$$
\left\langle g\left(\mathbf{k}-\mathbf{k}^{\prime}\right)^{2}\right\rangle_{\mathbf{k}^{\prime}}=\frac{\int_{-\pi}^{+\pi} d \phi_{\mathbf{k}^{\prime}} n\left(\mathbf{k}^{\prime}\right) g\left(\mathbf{k}-\mathbf{k}^{\prime}\right)^{2}}{\int_{-\pi}^{+\pi} d \phi_{\mathbf{k}^{\prime}} n\left(\mathbf{k}^{\prime}\right)}=g_{0}^{2},
$$

where $n\left(\mathbf{k}^{\prime}\right)=1 /\left|\nabla \epsilon\left(\mathbf{k}^{\prime}\right)\right|$ is the $L D O S$ and $g_{0}^{2}$ is independent of $q_{c}$. Such a normalization ensures that even if the modulation of $g(\mathbf{q})^{2}$ over the momentum transfer is governed by $q_{c}$, its $\mathbf{k}^{\prime}$-average over the Fermi surface remains equal to $g_{0}^{2}$. From the above condition, $g_{0}^{2}$, and so $\gamma\left(q_{c}\right)$, depends implicitly on the incoming electron momentum k. However in the following analysis we keep k fixed on the $Y$ point of the Fermi surface (see Fig.7) and we set $g_{0}^{2} /(4 t)=1$, as in the previous section.

From equations (9) and (10), it is clear that the structure in $\mathbf{q}$ of the $e-p h$ coupling $g(\mathbf{q})$ leads to a momentum dependence of the nonadiabatic corrections which cannot be described solely in terms of $\mathbf{q}=\mathbf{k}-\mathbf{k}^{\prime}$ for the vertex $P$ and $\mathbf{Q}=\mathbf{k}+\mathbf{k}^{\prime}$ for the cross $C$. For this reason, we employ the angular representation introduced in the previous section. This representation is also very useful for small $q$ ( and so small $\phi$ ) interactions which couples only nearby states for given point $\mathbf{k}$ in momentum space driving the electronic systems in the momentum decoupling $(M D)$ regime.21.22 When $M D$ is achieved for small values of the cutoff $q_{c}$ the anisotropy of several physical quantity such as the gap parameter, the tunneling conductance and the photoemission lineshape are driven by the LDOS.22 It is evident that $M D$ have to be relevant also for the $\mathbf{k}_{F}$ and $\mathbf{k}_{F}^{\prime}$ dependence of the vertex and cross corrections in the small $q$ limit.

In Fig. 9 we show the calculated vertex $P$ correction as a function of the angle $\phi$ for the optical phonon with momentum dependent coupling $g(\mathbf{q})$ for different values of the coupling cutoff $q_{c}=\pi / 16 ; \pi / 12 ; \pi / 8 ; \pi / 4$. The incoming electron momentum $\mathbf{k}$ has been chosen as in Fig.7 obtained with $t^{\prime}=0$ and $\mu /(4 t)=0.1$. The incoming and outcoming frequencies has been set equal to $\omega_{k}=\pi T$ and $\omega_{k^{\prime}}=\omega_{0}$, respectively, and as in the previous section the adiabatic parameter is $\omega_{0} /(4 t)=0.2$.

For all values of the momentum cutoff $q_{c}$, the vertex behaves in a way qualitatively similar to the one show in Fig. 8 for the structureless $e$ - $p h$ case: $P$ is positive for small values of $\phi$ and negative for large values. However, in the total pairing interaction $V\left(k, k^{\prime}\right)$ appearing in Eq.(可), the vertex $P\left(k, k^{\prime}\right)$ is multiplied by $g\left(\mathbf{k}-\mathbf{k}^{\prime}\right)^{2}$ which is peaked at small values of $\mathbf{k}-\mathbf{k}^{\prime}$ for small momentum cutoff $q_{c}$. As a consequence, $g\left(\mathbf{k}-\mathbf{k}^{\prime}\right)^{2}$ selects mainly the positive contributions of the vertex correction $P$. In the inset of Fig 9 we show the vertex correction for $q_{c}=\pi / 16$ evaluated at zero momentum transfer $(\phi=0)$ for different incoming electron running on the Fermi surface defined by the angle $\theta$; the maximum of the vertex has a weak dependence on $\theta$ and its anisotropy is driven by the LDOS. The effect is not strong but it is on the right direction with respect to the $M D$, giving the maximum correction near the saddle points where the $L D O S$ is high and the minimum near the $(\pi / 2, \pi / 2)$ point of the Brillouin zone where the $L D O S$ is low.

In Fig 10 we show the calculated cross correction $C$ for different values of the coupling cutoff $q_{c}=$ $\pi / 16 ; \pi / 12 ; \pi / 8 ; \pi / 4$. The angular dependence $\phi$ of $C$ is strongly sensitive to the cutoff value $q_{c}$. For $q_{c}=\pi / 4$ we recover a situation similar to the one obtained for momentum independent coupling: the relevant attractive contribution arises for large $\phi$ values $(\mathbf{Q} \simeq 0)$. When the $e$-ph coupling becomes peaked at small $\mathbf{q}$ the $\phi$ dependence of $C$ is completely different: the relevant attractive region moves from large to small $\phi$ values further increasing, together with the vertex, the total attractive correction for small momentum transfer. In the inset of Fig.10 we show the cross correction for $q_{c}=\pi / 16$ evaluated at $\mathbf{Q}=2 k_{F}(\phi=0)$ as a function of $\theta$. The small $\mathbf{q}$ coupling gives rise to strong anisotropy in the cross correction and near the saddle points is four times bigger than near the $(\pi / 2, \pi / 2)$ point. Then the cross correction increases the tendency to $M D$ already given by the momentum dependent small $\mathbf{q}$ coupling.

Finally we discuss the effect of small $\mathbf{q} e$-ph coupling on the effective pairing interaction $V\left(k, k^{\prime}\right)$, Eq.(6), keeping in mind the above results. In Fig.11, we report the pairing $V\left(k, k^{\prime}\right)$ in the angular $\phi$ representation (solid lines) for two different values of the momentum cutoff $q_{c}$ compared with the bare pairing $V_{0}\left(k-k^{\prime}\right)$ (dashed lines). For $q_{c}=\pi / 4$ (top panel) we find a slight pairing enhancing for small $\phi$ and a weak depression for large $\phi$; for $q_{c}=\pi / 16$ (bottom panel) we find a large amplification at small $\phi$ leading to an overall enhancement of the pairing. Therefore, by lowering the value of the momentum cutoff $q_{c}$ we expect that the nonadiabatic corrections enhances the averaged effective pairing. This situation is confirmed in Fig.12 where we plot the momentum average of $V\left(k, k^{\prime}\right)$ in units of the averaged bare interaction $V_{0}\left(k-k^{\prime}\right)$ as a function of the cutoff $q_{c}$ and for two different values of the outcoming frequency $\omega_{k^{\prime}}$. The averages are performed as described in Eq.(13), $\mathbf{k}$ being fixed on the $Y$ point of the Fermi surface. We find that, for frequency $\omega_{k^{\prime}}=\omega_{0},\langle V\rangle /\left\langle V_{0}\right\rangle$ is greater than unity for $q_{c}<\sim 0.5$ and that for a lower frequency $\left(\omega_{k^{\prime}}=0.3 \omega_{0}\right)$ the enhancement is found for a smaller value of $q_{c}$.

Summarizing, in this section we have shown how the e-ph coupling peaked at small momenta selects the attractive contribution of the nonadiabatic corrections giving an overall amplification of the pairing interaction. 


\section{CONCLUSIONS}

We have addressed the calculation of the vertex $P$ and the cross $C$ corrections to the Migdal-Eliashberg equations for specific band structures by numerical integration over the momentum space. We have considered a two-dimensional tight-binding model for the electron dispersion including near and next-near-neighbors hopping $\left(t, t^{\prime}\right)$ and an $e$-ph coupling in the nonadiabatic regime with an optical phonon of frequency $\omega_{0} /(4 t) \simeq 0.2$. Starting from a momentum independent $e$-ph coupling the $P$ and $C$ corrections provide a strong momentum and frequency dependence to the effective pairing. We find that the strenght of the pairing is enhanced for incoming electron momenta close to the outcoming ones due to the $P$ corrections and for almost opposite momenta due to the $C$ correction.

Considering a momentum dependent e-ph coupling, we find that small $\mathbf{q}$ peaked interactions select the attractive contribution of the vertex and cross terms leaving out the repulsive part, leading to an overall amplification of the total pairing.

Our main result is that the nonadiabatic vertex and cross corrections beyond Migdal's theorem lead to attractive contributions to the electron-electron interaction in the Cooper channel. These effects can provide a strong amplification of the superconducting critical temperature $T_{c}$. Moreover, the strong momentum dependence of the effective pairing induced by the nonadiabatic corrections can lead to strong anisotropy in the gap parameter and eventually to $d$-wave symmetry when a residual electron-electron repulsion is acting in the system.

\section{ACKNOWLEDGMENTS}

C. G. acknowledges the support of a I.N.F.M. PRA project.

${ }^{1}$ Y. J. Uemura et al., Phys. Rev. Lett. 66, 2665 (1991).

${ }^{2}$ N.M. Plakida, High temperature superconductivity: experiment and theory, Springer, Berlin (1995).

${ }^{3}$ O. Gunnarson, Rev. Mod. Phys. 69, 575 (1997).

${ }^{4}$ A.B. Migdal, Sov. Phys. JETP 34, 996 (1958).

${ }^{5}$ G. M. Eliashberg, Sov. Phys. JETP 11, 696 (1960).

${ }^{6}$ J.R. Schrieffer, J. Low Temp. Phys. 99, 377 (1995).

${ }^{7}$ C. Grimaldi, L. Pietronero and S. Strässler, Phys. Rev. Lett. 75, 1158 (1995).

${ }^{8}$ L. Pietronero, S. Strässler and C. Grimaldi, Phys. Rev. B 52, 10516 (1995); C. Grimaldi, L. Pietronero and S. Strässler, Phys. Rev. B 5210530 (1995).

${ }^{9}$ E. Cappelluti and L. Pietronero, Phys. Rev. B 53, 932 (1996); ibid., Europhys. Lett. 36, 619 (1996).

${ }^{10}$ C. Grimaldi, E. Cappelluti and L. Pietronero, cond-mat/9710159, preprint (1997).

${ }^{11}$ E. Cappelluti, C. Grimaldi, and L. Pietronero, cond-mat/9801232, preprint (1998).

${ }^{12}$ C. Grimaldi, L. Pietronero and M. Scattoni, preprint (1998).

13 M. R. Norman, M. Randeira, H. Ding, and J. C. Campuzano, Phys. Rev. B 52, 675 (1995).

${ }^{14}$ M. Mierzejewski, J. Zieliński, and P. Entel, Phys. Rev. B 57, 590 (1998)

${ }^{15}$ M. Grilli, and C. Castellani, Phys. Rev. B 50, 16880 (1994).

${ }^{16}$ C. Castellani, C. Di Castro, and M. Grilli, Phys. Rev. Lett. 75, 4650 (1995).

${ }^{17}$ R. Zeyher, and M. Kulic, Phys. Rev. B 53, 2850 (1996).

18 A. Perali, C. Castellani, C. Di Castro, and M. Grilli, Phys. Rev. B 54, 16216 (1996).

19 A. A. Abrikosov, Physica C 244, 243 (1995).

${ }^{20}$ M. Weger, M. Peter, and L. P. Pitaevskii, Z. Phys. B 101, 573 (1996).

${ }^{21}$ E. Sigmund, V.H. Hizhnyakov, D. Nevedrov, and A. Bill, J. of Superconductivity 10, 441 (1997).

${ }^{22}$ G. Varelogiannis, A. Perali, E. Cappelluti, and L. Pietronero, Phys. Rev. B 54, R6877 (1996). 

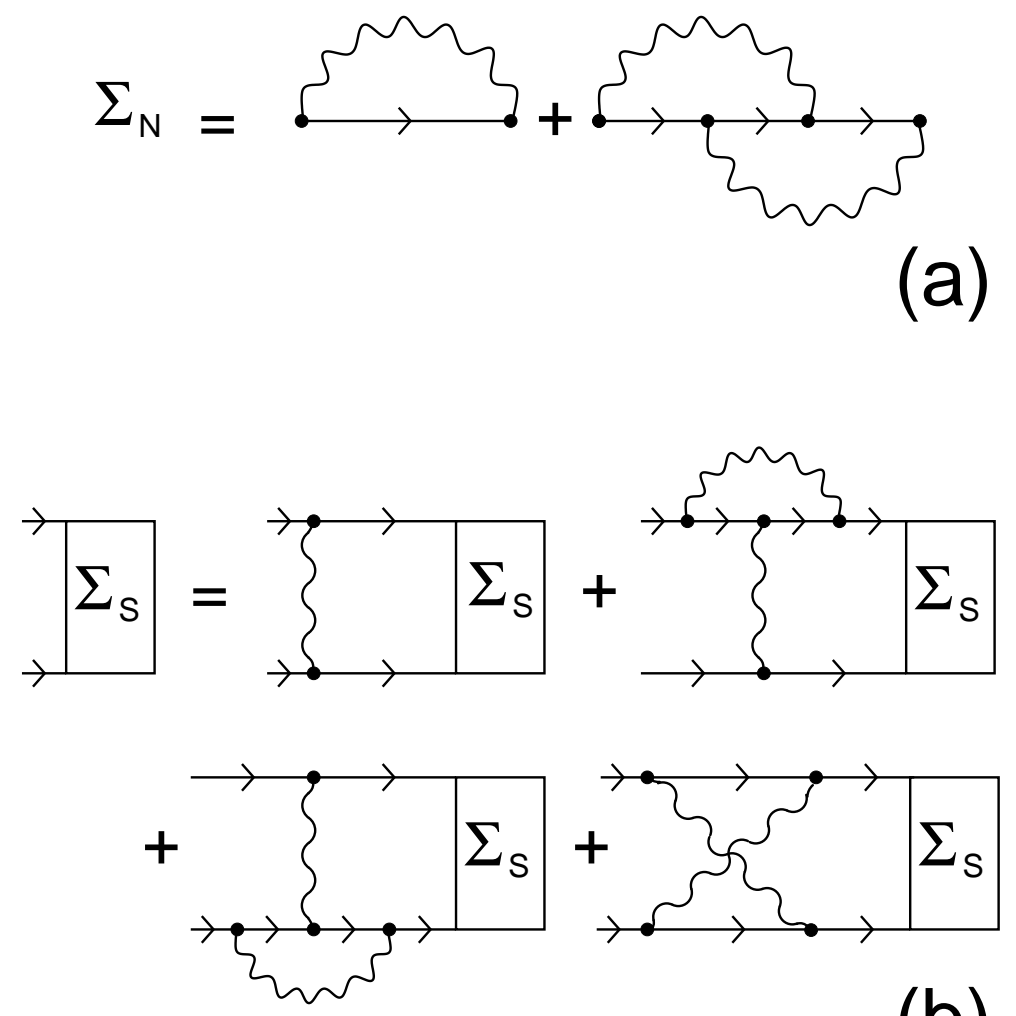

FIG. 1. (a): electron self-energy $\Sigma_{N}$ modified to include the first $e$-ph vertex correction (second diagram). (b): self-consistent equation for the off-diagonal electron self-energy. The ME theory retains only the first contribution while the first nonadiabatic corrections add the vertex (second and third diagrams) and the cross (last diagram) terms

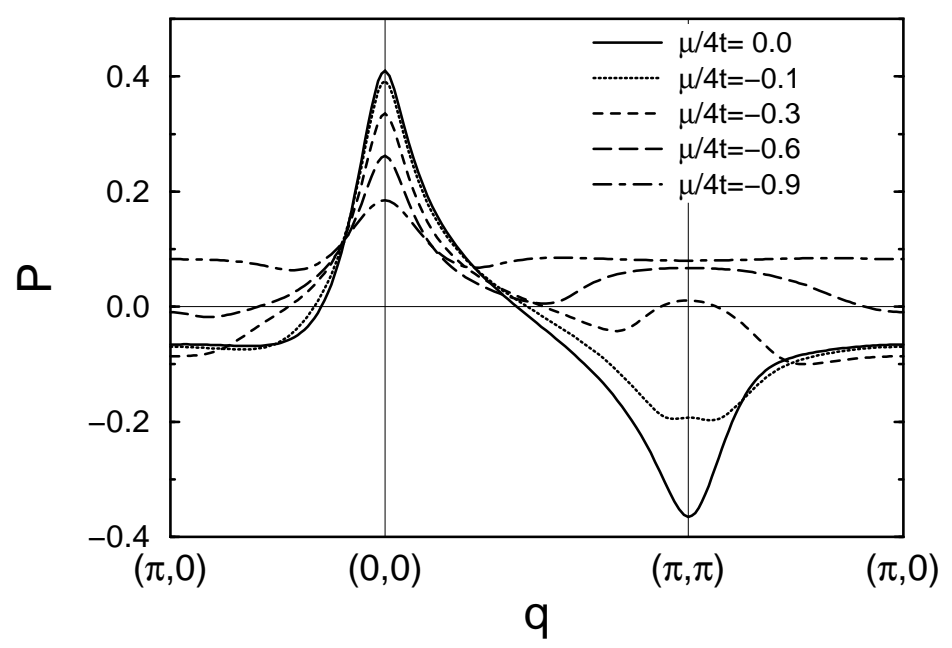

FIG. 2. Vertex function $P$ for momentum-independent electron-phonon coupling as a function of $\mathbf{q}=\mathbf{k}-\mathbf{k}^{\prime}$ and for different values of the chemical potential $\mu$. The calculation has been performed with $\omega_{0} /(4 t)=0.2, \omega_{k}=\pi T$ and $\omega_{k^{\prime}}=\omega_{0}$. 


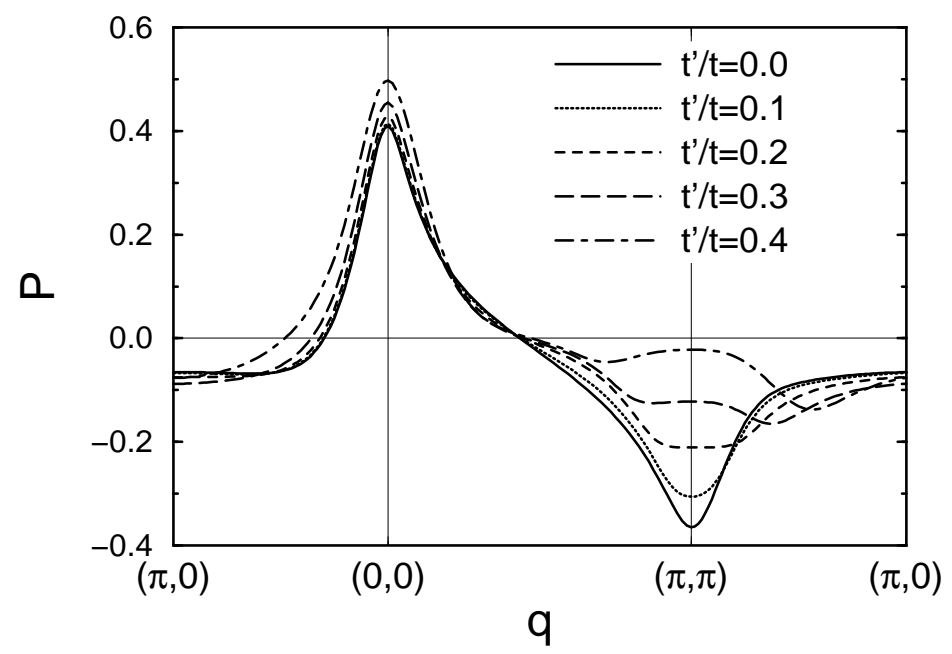

FIG. 3. Vertex function $P$ with $\mu=-4 t^{\prime}$ for different values of the next-nearest neighbor hopping parameter $t^{\prime} . \omega_{0} /(4 t)=0.2$, $\omega_{k}=\pi T$ and $\omega_{k^{\prime}}=\omega_{0}$.

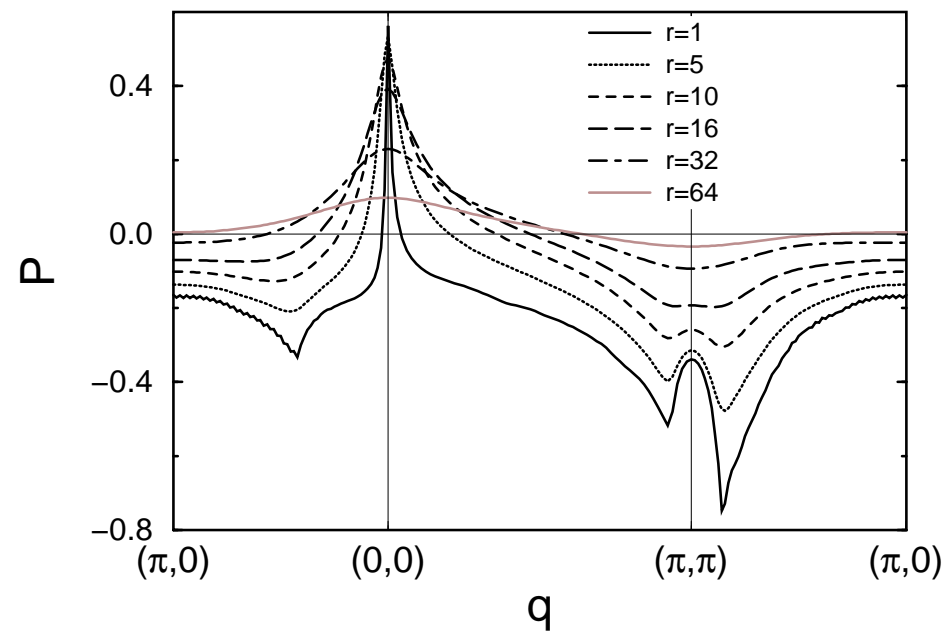

FIG. 4. Vertex function $P$ with $\mu /(4 t)=-0.1$ and $t^{\prime}=0$ for different values of the outgoing frequency $\omega_{k^{\prime}}$. $\omega_{0} /(4 t)=0.2$ and $\omega_{k}=\pi T$. 


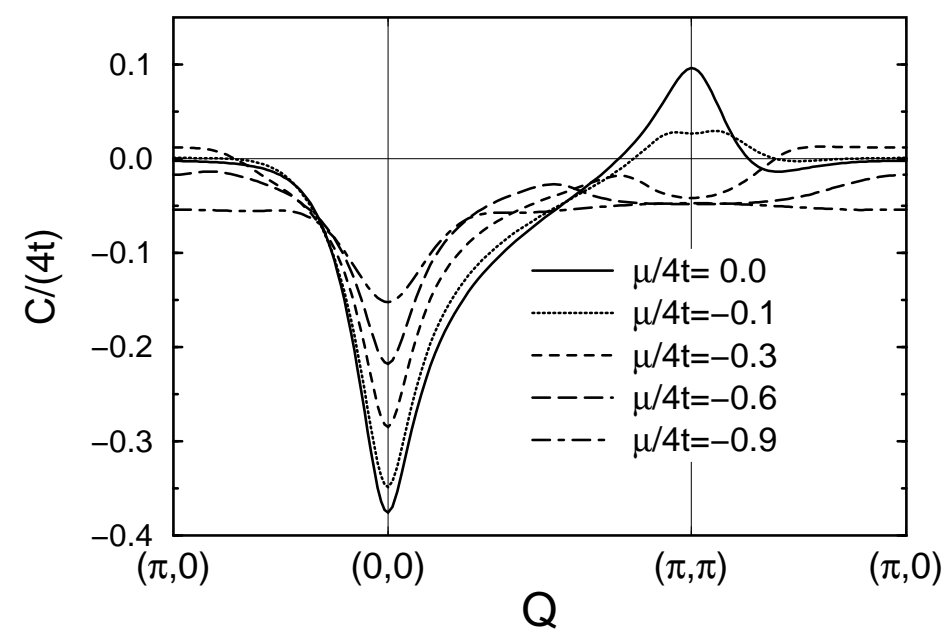

FIG. 5. Cross function $C$ for momentum-independent electron-phonon coupling as a function of $\mathbf{Q}=\mathbf{k}+\mathbf{k}^{\prime}$ and for different values of the chemical potential $\mu$. The calculation has been performed with $\omega_{0} /(4 t)=0.2, \omega_{k}=\pi T$ and $\omega_{k^{\prime}}=\omega_{0}$.

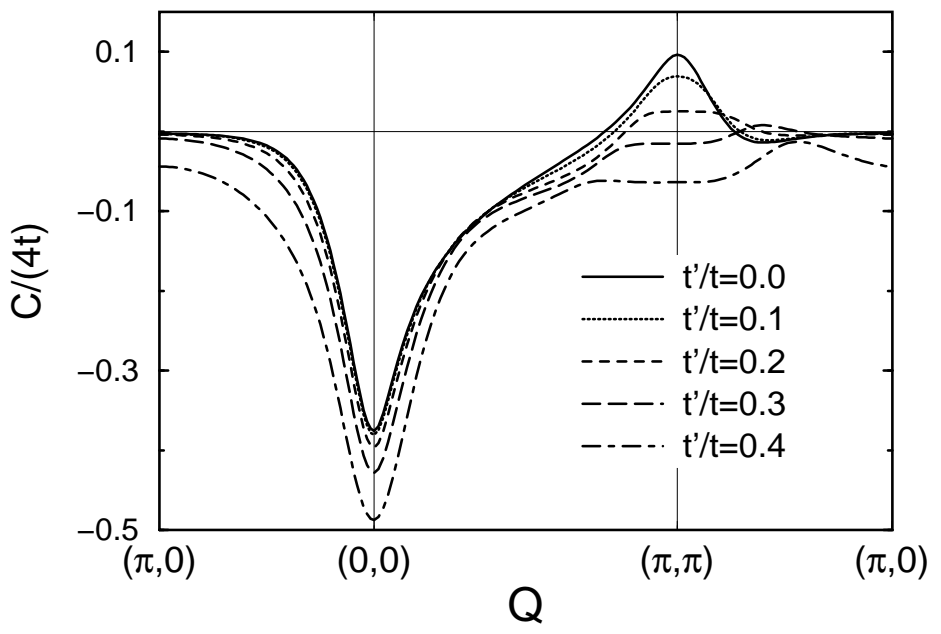

FIG. 6. Cross function $C$ with $\mu=-4 t^{\prime}$ for different values of the next-nearest neighbor hopping parameter $t^{\prime} . \omega_{0} /(4 t)=0.2$, $\omega_{k}=\pi T$ and $\omega_{k^{\prime}}=\omega_{0}$. 


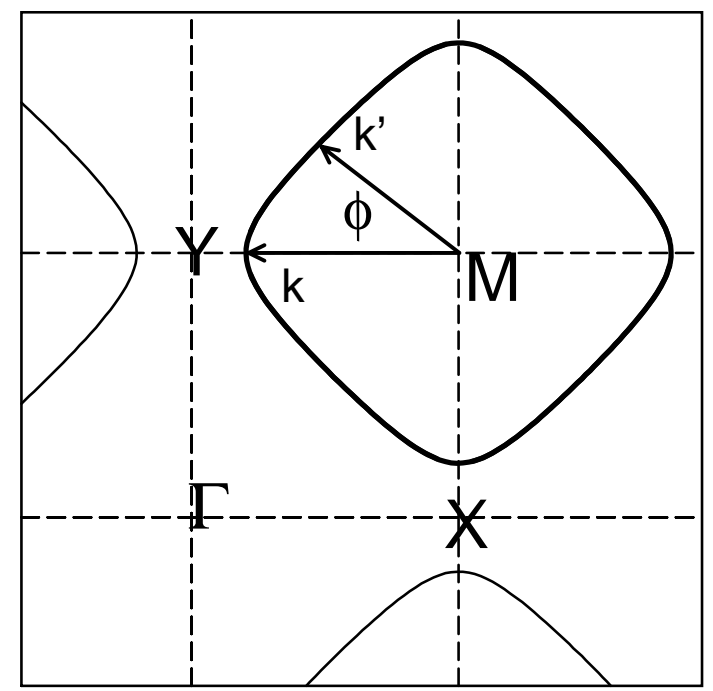

FIG. 7. Angular $\phi$-representation. The momentum $\mathbf{k}$ is fixed at one point of the Fermi surface and the angle $\phi$ measures the position of $\mathbf{k}^{\prime}$ with respect to $\mathbf{k}$.

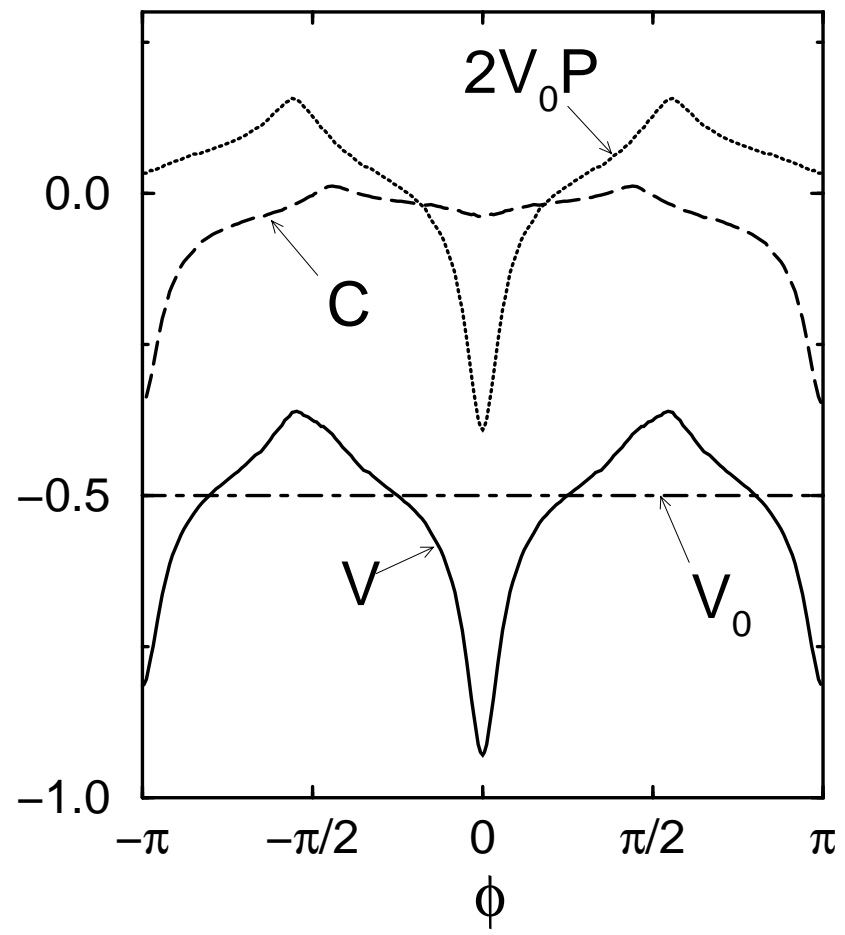

FIG. 8. Vertex $V$, cross $C$, bare $V_{0}$ and effective $V$ pairing interactions for momentum-independent electron-phonon coupling as a function of the angle $\phi$ between $\mathbf{k}_{F}$ and $\mathbf{k}_{F}^{\prime}$. All data are in units of $4 t$. The calculations are performed by setting $\omega_{k}=\pi T$ and $\omega_{k^{\prime}}=\omega_{0}$, so that the bare structureless interaction $V_{0}$ is close to -0.5 . 


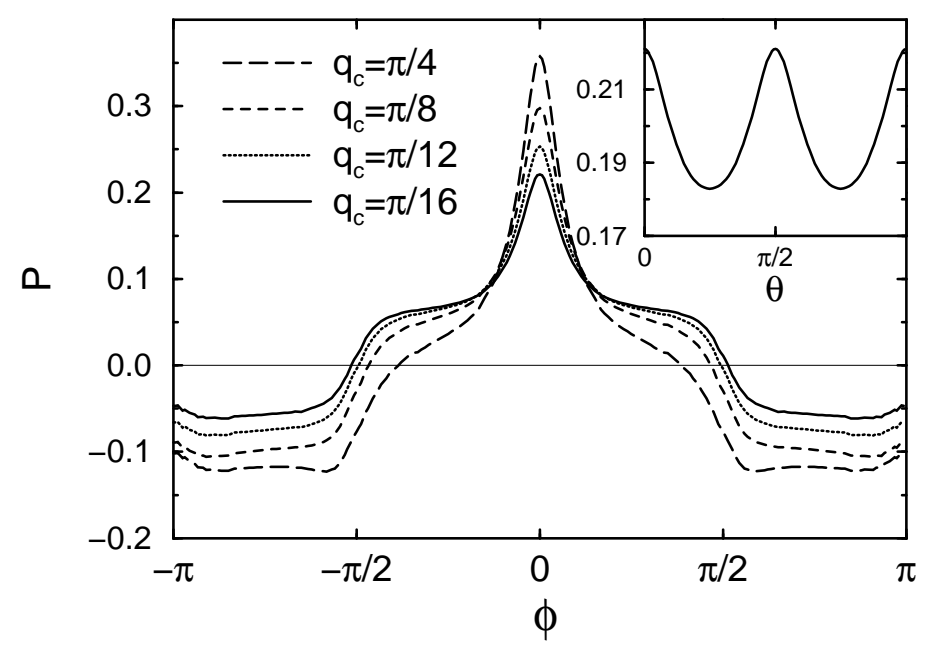

FIG. 9. Vertex function $P$ for momentum-dependent electron-phonon coupling in the angular representation (see text). $\omega_{0} /(4 t)=0.2, \omega_{k}=\pi T$ and $\omega_{k^{\prime}}=\omega_{0}$.

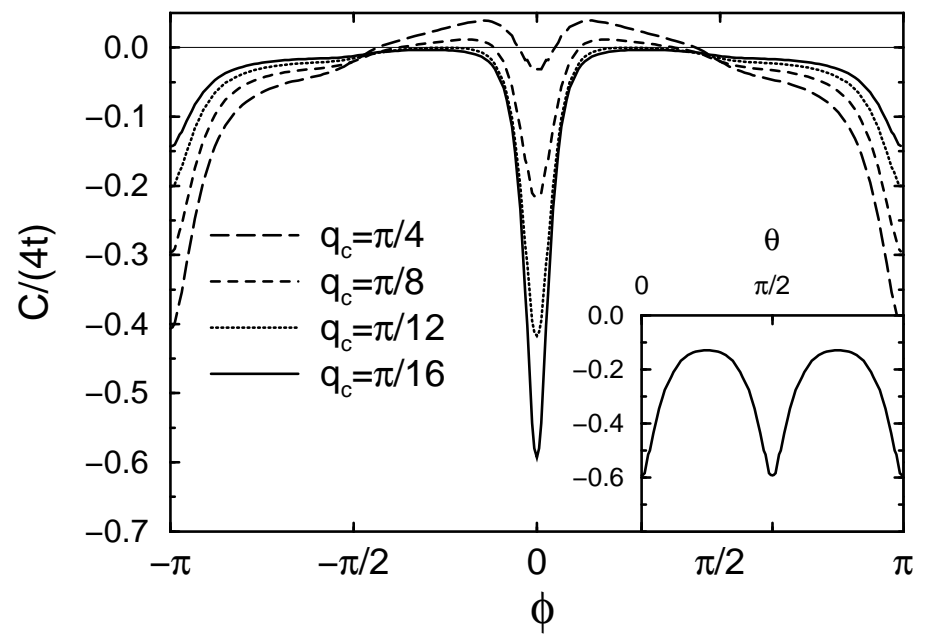

FIG. 10. Cross function $C$ for momentum-dependent electron-phonon coupling in the angular representation (see text). $\omega_{0} /(4 t)=0.2, \omega_{k}=\pi T$ and $\omega_{k^{\prime}}=\omega_{0}$. 


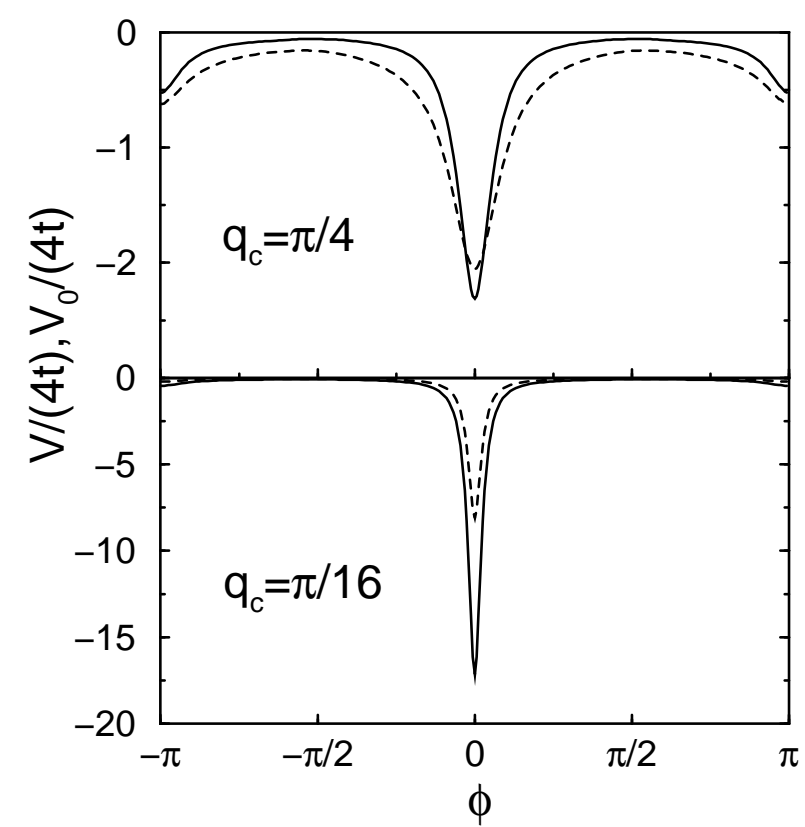

FIG. 11. Total pairing $V$ (solid lines) compared with the bare one $V_{0}$ (dashed lines) in the angular representation. $\omega_{0} /(4 t)=0.2, \omega_{k}=\pi T$ and $\omega_{k^{\prime}}=\omega_{0}$.

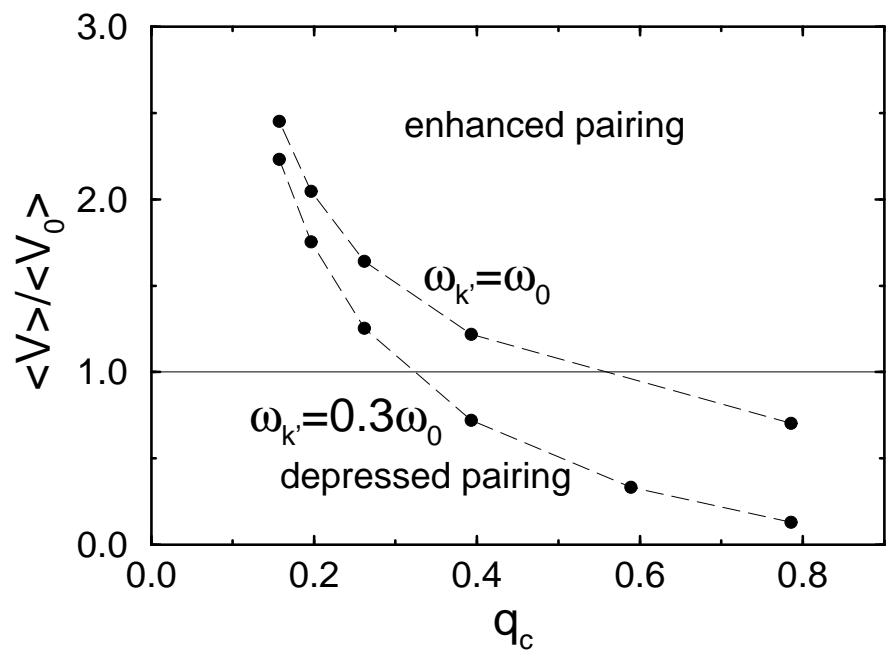

FIG. 12. Averaged total pairing normalized to the averaged bare interaction as a function of the momentum cutoff $q_{c}$ for two different outcoming frequencies. 